\title{
O desenvolvimento das tecnologias de informação e comunicação: efeitos na relação médico-paciente em Portugal
}

\author{
The development of information and communication technologies: \\ effects on the doctor-patient relationship in Portugal
}

\section{El desarrollo de las tecnologías de información y comunicación: efectos en la relación médico-paciente en Portugal}

\author{
Alexandre Morais Nunes ${ }^{1, a}$ \\ anunes@iscsp.ulisboa.pt | https://orcid.org/0000-0002-6808-7769
}

\begin{abstract}
${ }^{1}$ Universidade de Lisboa, Instituto Superior de Ciências Sociais e Políticas, Centro de Administração e Políticas Públicas. Lisboa, Portugal.
\end{abstract}

a Doutorado em Administração da Saúde pela Universidade de Lisboa.

\section{Resumo}

Nos últimos anos, em Portugal, a medicina apresentou elevado progresso com a integração das tecnologias de informação a serviço da saúde. No entanto, as novas tecnologias passaram também a fazer parte das rotinas dos pacientes, que cada vez estão mais informados sobre seus problemas de saúde. Este artigo procura identificar os impactos do recurso às tecnologias de informação e à internet na relação médicopaciente no sistema público de saúde. A pesquisa envolveu a aplicação de 100 entrevistas (50 feitas com médicos e 50 com usuários do sistema de saúde português). Como resultados, verificou-se que, apesar do desenvolvimento tecnológico e do crescimento do número de informações, a relação médico-paciente não está em risco, pelo fato de os pacientes continuarem confiando mais no seu médico do que na internet e acreditarem que, apesar de os sistemas de informação consumirem algum tempo da consulta, dão suporte para a decisão do médico a respeito do seu diagnóstico e tratamento dos pacientes.

Palavras-chave: Tecnologias da informação; Internet; Serviços de saúde; Relação médico-paciente; Confiabilidade.

\footnotetext{
Abstract

In recent years, in Portugal, medicine has made great progress with the integration of information technologies at the service of health. However, new technologies have also become part of the routine of patients which are more and more informed about their health problems. This article seeks to identify the impacts of the use of information technologies and of internet on the doctor-patient relationship. For the research, 100 persons (50 doctors and 50 users of the Portuguese public health system) were interviewed. The results showed that despite the technological development and the expansion of the information the doctor-patient relationship is not at risk, because patients continue to trust more their doctor than internet and they believe that although the information systems occupy some minutes of the physician consultation length, they give support to the doctor make his decisions about the diagnosis and the treatment of the patients.
} 
Keywords: Information technology; Internet; Health services; Doctor-patient relationship; Confidence.

\section{Resumen}

En los últimos años, en Portugal, la medicina ha avanzado con la integración de las tecnologías de la información al servicio de la salud. Sin embargo, las nuevas tecnologías también pasaron a formar parte de los costumbres de los pacientes que cada vez están más informados sobre sus problemas de salud. Este artículo busca identificar los impactos del recurso a las tecnologías de información e de la internet en la relación médico-paciente en el sistema público de salud. La investigación abarcó la aplicación de 100 entrevistas (50 realizadas con médicos y 50 con usuarios del sistema de salud portugués). Como resultados se verificó que, a pesar del desarrollo tecnológico y del crecimiento de la información, la relación médicopaciente no está en riesgo, por el hecho de que los pacientes continúan confiando más en su médico que en la internet y creen que, a pesar de los sistemas de información consumieren algún tiempo de la consulta, apoyan la decisión del médico acerca de su diagnóstico y del tratamiento de los pacientes.

Palabras clave: Tecnologías dela información; Internet; Servicios de salud; Relación médico-paciente; Confianza.

Contribuição dos autores:

Concepção e desenho do estudo: Alexandre Morais Nunes

Aquisição, análise ou interpretação dos dados: Alexandre Morais Nunes

Redação do manuscrito: Alexandre Morais Nunes

Revisão crítica do conteúdo intelectual: Alexandre Morais Nunes

Declaração de conflito de interesses: não há.

Fontes de financiamento: não houve.

Considerações éticas: não há.

Agradecimento/Contribuições adicionais: a todos os médicos e usuários que participaram nas entrevistas.

Histórico do artigo: Submetido: 02.nov.2017 | Aceito: 07.mar.2018 | Publicado: 29.jun.2018.

Apresentação anterior: não houve.

Licença CC BY-NC atribuição não comercial. Com essa licença é permitido acessar, baixar (download), copiar, imprimir, compartilhar, reutilizar e distribuir os artigos, desde que para uso não comercial e com a citação da fonte, conferindo os devidos créditos de autoria e menção à Reciis. Nesses casos, nenhuma permissão é necessária por parte dos autores ou dos editores.

\section{Introdução}

Nas últimas décadas, a medicina evoluiu do ponto de vista científico assim como no que diz respeito ao acesso ao conhecimento, o que permitiu novos conhecimentos teóricos sobre doenças emergentes e seus efeitos ${ }^{1}$.

Acompanhando esse progresso, esteve presente um crescimento contínuo das tecnologias e dos sistemas de informação, que melhoraram a transmissão de informações entre médicos e outros profissionais de saúde e permitiram também uma maior e mais célere comunicação com a adoção de um registro clínico eletrônico².

Em Portugal, o desenvolvimento das tecnologias e sistemas de informação em saúde foi além disso, e foram desenvolvidos inúmeros aplicativos que facilitam o trabalho dos profissionais de saúde e permitem uma maior monitorização do sistema de saúde. 
Outro ponto importante dos efeitos do desenvolvimento das tecnologias de informação foi o crescimento do acesso da população à internet para consulta de informações de saúde e bem-estar. Hoje em dia, é imensa a quantidade de informação médica e de saúde que se encontra à disposição dos pacientes ${ }^{3-4}$. Assim, cada vez mais, por meio da internet, os pacientes têm acesso a comunidades virtuais, fóruns de discussão, chats e trocas de e-mails com informações sobre saúde. De acordo com estudo realizado por Santana e Pereira $^{5}$, 26\% dos usuários de internet recorrem a esse meio para obterem informação que ajude a decidir se devem ou não consultar um profissional de saúde, 25\% para obterem informação sobre saúde antes de uma consulta e 25,6\% para obterem informação depois de uma consulta com profissionais de saúde.

Há várias razões que levam os pacientes a procurarem informações sobre saúde na internet. De acordo com a literatura, os usuários são guiados simplesmente pela curiosidade, pelos seus problemas de saúde, pela existência de dificuldades em contatar seu médico, para tentar autodiagnosticar um problema de saúde associado aos sintomas de desconforto ou apenas para pesquisar informação relacionada com a promoção da saúde e hábitos de vida saudáveis ${ }^{6}$.

A busca de informação de saúde através da internet é um novo desafio que tem os seus benefícios educacionais e que demonstra uma preocupação dos pacientes pelo seu próprio estado de saúde ${ }^{7}$ Contudo, é necessário ter sempre presente que existem fontes de informação pouco críveis que podem conduzir ao erro $^{8-9}$ e até poderão, de acordo com a revisão da literatura, influenciar a relação entre o médico e o paciente. Por um lado, o uso de tecnologias de informação no decorrer da consulta pode levar os médicos a focarem a sua atenção no computador perdendo parte da humanização no atendimento ${ }^{10}$ e, por outro, a informação previamente obtida pelo paciente na internet poderá criar um confronto com a informação dada pelo médico, levando muitas vezes a uma quebra de confiança.

Este artigo tem como objetivo identificar o impacto do uso das tecnologias de informação e comunicação nas relações humanas entre pacientes e médicos do sistema público de saúde em Portugal, de forma a apurar a realidade sentida pelos pacientes relativamente ao seu médico e também a verificar o grau de consulta de informação pela internet e de que forma isso pode influenciar o seu comportamento.

\section{A utilização das tecnologias de informação e comunicação por médicos}

Como dito anteriormente, o uso de tecnologias de informação e comunicação é, cada vez mais, realizado por profissionais de saúde e pacientes.

O desenvolvimento das tecnologias e sistemas de informação em saúde levou ao desenvolvimento de vários aplicativos que auxiliam o trabalho dos profissionais de saúde, facilitando o acesso ao processo e a registros clínicos, a prescrição e o acesso aos resultados dos exames realizados ${ }^{2-10}$.

No caso português, nos últimos 10 anos foram dados importantes passos na informatização do setor da saúde, com a criação de programas informáticos inovadores como:

- A Plataforma de Dados da Saúde (PDS): sistema que permite o registro e o compartilhamento de informação clínica entre o paciente, os profissionais de saúde e as entidades prestadoras de serviços, de acordo com os requisitos da Comissão Nacional de Proteção de Dados (CNPD);

- A Prescrição Eletrônica Médica: permite a rápida prescrição de medicamentos de acordo com a Denominação Comum Internacional (DCI), por via eletrônica e sustentada por normas de orientação clínica, e a desmaterialização dos procedimentos associados ao circuito de prescrição - dispensa - faturação - conferência, para garantir maior eficiência e segurança no circuito do medicamento;

- O SClínico Cuidados de Saúde Primários (CSP): sistema de informação que nasceu da vasta experiência com dois programas anteriores usados por milhares de médicos, enfermeiros e outros técnicos de saúde: o Sistema de Apoio ao Médico (SAM) e o Sistema de Apoio à Prática de 
Enfermagem (SAPE). Essa ferramenta foi adotada por mais de 300 instituições, sendo utilizada por mais de 13.000 profissionais;

- O SClínico Hospitalar: sistema de informação que nasceu da vasta experiência com dois programas anteriores, usados por milhares de médicos, enfermeiros e outros técnicos de saúde: o SAM e o SAPE. Essa ferramenta está presente em mais de 50 Centros Hospitalares, Misericórdias e Institutos, e conta com cerca de 60.000 profissionais registados.

- O SI.VIDA: sistema de informação que permite a monitorização e o acompanhamento de usuários com HIV/Aids; "é a ferramenta de suporte à monitorização do Programa, com o propósito de registar toda a atividade realizada no âmbito da prestação destes cuidados" ${ }^{” 1}$.

- O Sistema de Informação dos Certificados de Óbito (SICO): sistema de informação cuja finalidade é permitir uma articulação das entidades envolvidas no processo de certificação dos óbitos, com vista a promover uma adequada utilização dos recursos, a melhoria da qualidade e do rigor da informação, e a rapidez de acesso aos dados em condições de segurança e de respeito à privacidade dos cidadãos.

- O Sistema Nacional de Apoio à Vigilância Epidemiológica (SINAVE): foi criado para implementar um sistema informático que desmaterialize "o processo de vigilância epidemiológica das doenças de declaração obrigatória e permita o registro das notificações e dos inquéritos epidemiológicos, respetivamente pelos médicos notificadores e delegados de saúde pública, locais e regionais. Permite, também, a produção de estatísticas para a Direção-Geral de Saúde (DGS), recorrendo ao sistema de referência geográfica ArcGis, e para reporte ao ECDC (European Committee for Disease Control)"12.

Essas ferramentas informáticas são fundamentais para melhorar o atendimento ao paciente, que deve ser sempre o principal foco de ação do trabalho do médico. A centralização da atividade no paciente já é uma discussão que, na história da medicina, tem vários anos. De acordo com Jaspers ${ }^{13} \mathrm{e}$ com Coelho Filho ${ }^{14}$, a comunicação entre médico e paciente durante uma consulta é fundamental. Essa focalização promove a humanização no atendimento, o que é um marco importante para melhoria da qualidade, da confiança e da satisfação do consulente que respeitará o aconselhamento dado ${ }^{15-16}$.

\section{A utilização das tecnologias de informação e comunicação (via internet) por pacientes}

Quanto aos pacientes, em Portugal, tem-se assistido a um crescimento da utilização da internet (mais de $73 \%$ de usuários diários) ${ }^{17}$.

Cada vez mais, a internet é usada como fonte de informação sobre a saúde e leva os pacientes a obterem mais informações sobre os seus problemas. Com o crescimento do acesso à internet, eles têm cada vez mais necessidade de pesquisar casos semelhantes aos seus antes de irem ao consultório ${ }^{18}$. De acordo com Kim e Kim ${ }^{19}$, no mundo existem diariamente milhões de buscas de informações relacionadas à saúde e absorvidas pelos pacientes independentemente da opinião do médico. Em Portugal, num estudo realizado por Santana e Pereira ${ }^{20}$, os cidadãos admitem mesmo que recorrem à internet para avaliar a necessidade de realização de consulta ou, ainda, para obter informação depois da consulta e confrontá-la com a opinião ou o diagnóstico do médico por causa da insatisfação com as recomendações dos profissionais.

O contato com outros pacientes e o interesse do cidadão em procurar informações de saúde na internet podem estar relacionados com um problema no seu estado de saúde ou no seu estilo de vida, que o deixem em situação de risco, e ainda constituem uma oportunidade para trocar experiências ${ }^{21}$. Por outro lado, e de acordo com Masi, Cassey, Suareaz-Balcazar, Cassey, Kinney e Piotrowski ${ }^{22}$, mais informação dos pacientes sobre as suas doenças contribui para a redução dos custos com a prestação de cuidados de saúde, pois os 
cidadãos estão mais alertados e recorrem ao médico ao primeiro sinal de agravamento do seu estado de saúde.

No entanto, junto dos benefícios da pesquisa de informações através da internet é preciso conjugar algumas preocupações relacionadas com a qualidade dos conteúdos disponibilizados ${ }^{7-19}$. Por vezes, existe informação falsa que pode pôr em risco a relação médico-paciente, pela perda de credibilidade do médico perante a informação coletada na internet ${ }^{23-24}$.

\section{Metodologia de pesquisa}

Para verificar os efeitos da utilização de tecnologias e sistemas de informação, em Portugal, na relação médico-paciente foram realizadas 100 entrevistas no Distrito de Castelo Branco. Foram estudadas duas populações: a dos médicos (50 inquiridos com idade compreendida entre os 30 e os 65 anos com mais de 10 anos de trabalho no sistema público de saúde) e a dos pacientes do sistema de saúde (50 inquiridos com idades compreendidas entre os 18 e os 65 anos que utilizam a atenção primária e hospitalar do sistema público). Apenas dessa forma seria possível estudar o tema num todo, verificando ambas as perspectivas.

As entrevistas foram realizadas em língua portuguesa, e as questões foram colocadas tendo por base a revisão da literatura realizada. Para os médicos, foi perguntado:

1. A adoção dos novos sistemas e tecnologias de informação prejudicou o tempo de atendimento do paciente?

2. Considera uma vantagem para a saúde a consulta de informação na internet?

3. A busca de informação sobre saúde na internet pode transformar a relação médico-doente?

4. Teve conflito com algum paciente por ele ter seguido recomendações da internet e não as que solicitou?

5. A desprofissionalização do trabalho médico pode ocorrer no futuro?

Para os pacientes, foram colocadas as seguintes questões:

1. Considera que o seu médico dá mais atenção ao computador do que a você durante a consulta?

2. Entende que os novos sistemas de informação são uma vantagem para o seu médico conhecer melhor o seu estado de saúde?

3. A informação na internet é útil e vantajosa para a sua saúde?

4. Já buscou informação de saúde na internet e confrontou seu médico com ela? Se sim, qual a reação dele?

Foi garantido o anonimato dos médicos e dos pacientes inquiridos. Os dados foram tratados por meio da análise de conteúdo ${ }^{25}$, técnica que, associada com as respostas às questões de uma entrevista, permite decodificar a informação organizando-a em categorias.

\section{Resultados}

Usando a técnica da análise de conteúdo, as respostas foram operacionalizadas e organizadas de acordo com a opinião dos entrevistados.

\section{Resultados relativos às entrevistas realizadas com os médicos}

No geral, 35 médicos (70\%) discordam da ideia de que as novas tecnologias tenham prejudicado o tempo de atendimento, porque antes teriam de consultar manualmente os processos dos pacientes em papel e 
escritos por outros colegas, o que dificultava o entendimento. Como exemplos, os médicos entrevistados respondem:

\begin{abstract}
"a informação eletrônica facilita a pesquisa e com ela conhecemos melhor o paciente, que fica satisfeito por conhecermos toda a sua ficha clínica" (Médico 2).

"Tenho 1.8oo pacientes na minha listagem; com os programas online, consegui visualizar rapidamente os problemas do paciente e a medicação que ele toma" (Médico 15).

"As novas tecnologias serviram para facilitar os procedimentos e ganhamos tempo na procura da informação e na interpretação da caligrafia de outros colegas que, por vezes, não se entende" (Médico 34).
\end{abstract}

Ainda relativamente ao exposto nessa questão, 15 médicos (30\%) relatam que, às vezes, os sistemas de informação não funcionam ou são demasiado lentos, o que atrasa as consultas e deixa o paciente insatisfeito.

Todos os médicos alegam que não há qualquer vantagem para o paciente que consulta informação na internet e 30 médicos (60\%) acreditam que essa informação pode transformar a sua relação com seus pacientes, pois consideram que

"os pacientes confiam plenamente no seu médico, mas por vezes pode gerar conflito" (Médico 33).

"A informação na internet vale o que vale, poderá ser sempre falsa" (Médico 38).

Apenas seis médicos (12\%) relatam que tiveram conflitos com pacientes relacionados com informações de saúde coletadas na internet. No complemento da resposta, destacaram que esses conflitos foram ocasionados com pacientes jovens e do sexo feminino.

Todos os médicos entrevistados negam que esteja em jogo a desprofissionalização do trabalho médico, referindo que

“a imagem do médico em Portugal prevalecerá à informação muitas vezes anônima” (Médico 12).

"Não tenho qualquer dúvida que nunca ocorrerá a desprofissionalização médica" (Médico 44).

\title{
Resultados relativos às entrevistas realizadas com os pacientes
}

Na opinião de 40 pacientes (80\%), o médico dá mais atenção ao computador nele fixando o seu olhar e se esquece do seu paciente.

"Estamos ali esperando que o doutor veja o computador e, no final, ele diz que está tudo bem e dá o receituário" (Paciente 9).

"Mais de 9o\% do tempo de consulta é entre o doutor e o computador, o resto é para nós pacientes" (Paciente 45).

"O médico pouco fala para nós, ele vai falando para o computador que parece estar sempre com problemas de conexão" (Paciente 34).

Embora afirmando que o médico passa a maior parte do tempo olhando para o computador, todos os pacientes entrevistados (100\%) consideram que os sistemas de informação permitem consultar rapidamente o seu processo clínico e evitam que se tenha de levar resultados de exames e outros documentos. Sobre esta questão foi relatado:

“o computador tem lá tudo, não precisamos de levar nada em papel” (Paciente 27). 
"Antes o doutor tinha papel amarelo em cima da mesa, agora não, está tudo nas máquinas" (Paciente 50).

"Sempre que preciso de um documento sobre o meu processo, o doutor tem lá no computador" (Paciente 2).

36 dos 50 pacientes entrevistados (72\%) reconhecem que a informação da internet é útil para a promoção da sua saúde. Os restantes desvalorizam e afirmam ter várias dúvidas quanto à veracidade:

"Não confio na informação da internet, prefiro perguntar ao meu médico"(Paciente 19).

"Na internet cada um escreve o que quer" (Paciente 41).

No entanto, apesar da opinião da maioria, nenhum dos pacientes confrontou o seu médico com informação encontrada na internet e considera esse enfrentamento uma falta de respeito:

“o doutor é médico e, por isso, sabe o que diz” (Paciente 33).

"Prefiro seguir as recomendações do meu médico que a dos estranhos" (Paciente 5).

Em resumo, os resultados das entrevistas realizadas se apresentam nas tabelas seguintes:

Tabela 1 - Impacto das tecnologias de informação e comunicação na relação médico-paciente na perspectiva do médico

\begin{tabular}{|l|l|l|}
\hline & Sim & Não \\
\hline $\begin{array}{l}\text { A adoção dos novos sistemas e tecnologias de informação prejudicou o tempo de atendimento } \\
\text { do paciente? }\end{array}$ & $30 \%$ & $70 \%$ \\
\hline Considera uma vantagem para a saúde a consulta de informação na internet? & $0 \%$ & $100 \%$ \\
\hline A busca de informação sobre saúde na internet pode transformar a relação médico-doente? & $40 \%$ & $60 \%$ \\
\hline $\begin{array}{l}\text { Teve conflito com algum paciente por ele ter seguido recomendações da internet e não as que } \\
\text { solicitou? }\end{array}$ & $6 \%$ & $94 \%$ \\
\hline A desprofissionalização do trabalho médico pode ocorrer no futuro? & $0 \%$ & $100 \%$ \\
\hline
\end{tabular}

Fonte: O autor (2018).

Tabela 2 - Impacto das tecnologias de informação e comunicação na relação médico-paciente na perspectiva do paciente

\begin{tabular}{|l|l|l|}
\hline & Sim & Não \\
\hline Considera que o seu médico dá mais atenção ao computador do que a você durante a consulta? & $80 \%$ & $20 \%$ \\
\hline $\begin{array}{l}\text { Entende que os novos sistemas de informação são uma vantagem para o seu médico } \\
\text { conhecer melhor o seu estado de saúde? }\end{array}$ & $100 \%$ & $0 \%$ \\
\hline A informação na internet é útil e vantajosa para a sua saúde? & $72 \%$ & $28 \%$ \\
\hline $\begin{array}{l}\text { Já buscou informação de saúde na internet e confrontou seu médico com ela? Se sim, qual } \\
\text { a reação? }\end{array}$ & $0 \%$ & $100 \%$ \\
\hline
\end{tabular}

Fonte: O autor (2018).

\section{Discussão}


Este estudo examinou a influência dos sistemas de informação e da internet sobre a relação médicopaciente, numa amostra de médicos do Serviço Nacional de Saúde de Portugal e de pacientes da assistência médica básica na região de Castelo Branco.

No questionário realizado com os médicos, encontrou-se uma percepção positiva relativamente às vantagens das novas tecnologias de informação e comunicação, que podem contribuir positivamente para um melhor atendimento do paciente. Além disso, os pacientes inquiridos alegaram que os sistemas de informação são uma vantagem, pois o médico tem acesso rápido a todas as informações. Essa interpretação vai ao encontro da literatura. Goodman ${ }^{16}$ estudou este tema e colocou questões semelhantes para os médicos, com o objetivo de analisar os efeitos das tecnologias de informação, concluindo que o médico não perdeu seu conhecimento nem a sua relação com o paciente e, pelo contrário, tem uma maior capacidade de decisão um maior apoio para ela. Também o estudo de Hummel ${ }^{9}$ tirou conclusões idênticas, referindo que as tecnologias de informação e comunicação tornaram-se determinantes para o funcionamento e desenvolvimento de qualquer organização, na medida em que facilitaram processos, promoveram a inovação e facilitaram a comunicação. Atualmente, é mesmo inconcebível imaginar uma instituição de saúde sem a utilização de tecnologias de informação $0^{26}$.

Vários pesquisadores têm estudado este assunto, entre os quais se destacam os trabalhos de: Macgregor, Hyland e Harvie ${ }^{27}$, que concluíram que a internet, aliada às tecnologias de informação, permitiu explorar as novas tecnologias e melhorar o atendimento nos hospitais, a eficácia na comunicação entre profissionais de saúde e a transmissão de informação ao paciente; El-Sayed e Westrup ${ }^{28}$, que concluíram que o recurso a tecnologias de informação e comunicação nos hospitais contribuíram para a melhoria das práticas de comunicação, tornando o sistema de atendimento ao paciente mais eficiente; e os de Akesson, Saveman e Nilssom $^{29}$, de acordo com os quais a melhoria na qualidade do atendimento nas unidades e da disponibilização da informação se deveu à adoção de tecnologias e sistemas de informação. Outros autores, como Seckin ${ }^{30}$ e Hummel 9 , foram mais longe nos seus trabalhos, revelando que, além da melhoria da comunicação e interação com os pacientes, as tecnologias de informação e comunicação melhoraram as interações entre os profissionais de saúde.

Os pacientes relatam que o médico perde mais tempo com o computador que com o próprio paciente, comprometendo o seu relacionamento. Essa situação não é encontrada na revisão da literatura, em que se destaca Salles ${ }^{31}$. Nas conclusões do seu trabalho, esse autor considerou a informatização da prática da medicina uma mais-valia para a saúde, permitindo uma maior rapidez e eficiência como as encontradas na consulta dos processos, o que dá mais tempo para a relação médico-paciente.

Os pacientes entrevistados consideraram útil a informação sobre saúde disponível na internet e afirmam recorrer a essa consulta quando necessário. Sobre esta questão, a literatura compartilha da mesma opinião e defende que o acesso à informação científica é cada vez mais utilizado pelos cidadãos, que veem na internet uma forma fácil de acessar essa informação e procuram dados sobre a sua doença, sintomas e medicamentos ${ }^{32}$. Contudo, os médicos entrevistados desvalorizaram as vantagens dessas informações, alegando que, por vezes, são falsas e não correspondem à verdade, o que pode causar um mal-estar no consultório. Essa perspectiva é também confirmada pela literatura, como ilustram os trabalhos de Grosseman e Stoll ${ }^{33}$, ao relatarem que as múltiplas informações obtidas na internet podem interferir na relação dos médicos com seus pacientes.

Outros estudos com metodologia semelhante foram realizados por pesquisadores norte- americanos. Como exemplos, em seus trabalhos, Budtz e Witt ${ }^{34}$ entrevistaram 93 pacientes e concluíram que apenas 18 recorriam à internet para pesquisa de informação sobre a sua saúde; Grover, Blanford, Holcomb e Tidler ${ }^{35}$, numa amostra de 127 pacientes no Colorado, verificaram que 40 já haviam usado a internet para consultar informações sobre a sua saúde e Diaz, Griffith, Ng, Reinert, Friedman e Moulton ${ }^{36}$, numa amostra de 512 pacientes, comprovaram que 204 acediam à internet para melhor se informarem sobre os seus problemas de 
saúde. Na Europa, destaca-se um estudo realizado por Andreassen, Bujnowska-Fedak, Chronaki, Dumitru, Pudele, Santa et al. ${ }^{37}$, que entrevistaram oito mil pacientes em sete países e concluíram que apenas 2.386 acediam à internet para buscas relacionadas com a sua saúde.

Apesar dos médicos entrevistados não registrarem confrontos com os seus pacientes, a literatura considera que esse evento poderá ocorrer no futuro, uma vez que os pacientes estão progressivamente munidos de informação e deixam de ter uma confiança 'cega' no seu médico, questionando-o cada vez mais. De acordo com um estudo realizado por Fox e Renie ${ }^{38}$, de 500 pacientes 190 confirmaram ter confrontado os seus médicos com informações da internet.

Sobre a questão da desprofissionalização, os médicos entrevistados discordam dessa ideia e consideram que ela sequer esteja em risco, negando a sua existência. Essa opinião é confirmada na literatura. Como exemplo, tem-se o trabalho de Henwood, Hart e Smith ${ }^{39}$, por meio do qual concluíram que o maior conhecimento dos cidadãos sobre a sua saúde não implica uma perda da confiança ou autoridade do médico, há apenas uma troca de informações, o que é saudável para a relação médico-paciente. Pandey Hart e Tiwary40 constataram que, apesar das informações obtidas por um grupo de mulheres na internet, a confiança que elas tinham em seus médicos superou qualquer informação. Os trabalhos de Skinner, Biscope e Poland ${ }^{41}$, realizados com uma população mais jovem, levaram aos mesmos resultados, entendendo o médico como um colaborador e a internet como uma fonte de informação que gera dúvidas. Nas suas conclusões, Cottem e Gupta ${ }^{42}$ apontam a internet como uma fonte de informação complementar à informação de primeira linha transmitida pelo médico. De acordo com os resultados dos estudos de Berger, Wagner e Baker ${ }^{43}$ e de Nettleton, Burrows e O’Malley ${ }^{44}$, a informação da internet é saudável porque levou os cidadãos a procurarem ajuda médica e a recusarem a informação da internet sem antes consultar o seu médico, o que revela uma maior confiança na relação médico-paciente.

$\mathrm{Na}$ literatura, essa opinião não é consensual e existem autores que acreditam que a assimilação de informações, por parte dos pacientes, pode contribuir para a desprofissionalização médica. Como exemplos, podem ser citados os trabalhos de Hardey ${ }^{45}$ que, numa pesquisa realizada no Reino Unido, concluiu que, anteriormente, os médicos exerciam o seu poder pelo fato de deterem o conhecimento sobre a saúde, mas que a divulgação da informação pela internet reduziu essa confiança e promoveu a busca de alternativas. Também Dentrea e Moren-Cross ${ }^{46}$ assim como Fox, Ward, O’Rourke ${ }^{47}$, ao estudarem essa problemática, concluíram que os sites de informação sobre saúde consolidam os conhecimentos dos pacientes e reduziram as visitas ao médico, contribuindo dessa forma para a desprofissionalização. Para Ziebland ${ }^{48}$, no Reino Unido, o acesso às informações, antes reservadas aos médicos, pode reduzir a autoridade e o respeito pelo médico, contribuindo assim para a desprofissionalização.

Pelo exposto, Henwood, Hart e Smith ${ }^{39}$ recomendam que é fundamental que os médicos procurem dar mais atenção ao paciente, escutando-o, valorizando suas queixas e fornecendo informações de qualidade, discutindo os resultados dos exames e os diagnósticos com o apoio dos sistemas de informação.

\section{Considerações finais}

O desenvolvimento de sistemas de informação de apoio à prática clínica em Portugal aumentou na última década, tal como o uso da internet pelos pacientes para obterem informações sobre questões de saúde. Como resultados, verificou-se que, para os pacientes, um crescimento dos sistemas e tecnologias de informação é uma medida positiva que permite ao médico um maior conhecimento do seu registro de saúde, mas também desfoca a sua atenção e faz perder tempo de diálogo. Por outro lado, verificou-se que a internet é uma fonte de informação importante para o paciente motivado pelo interesse pessoal em saber mais sobre o seu problema, mas em nada põe em risco a sua relação com o médico, uma vez que a informação dada por este é considerada como a mais confiável. 
Pelo trabalho realizado, concluiu-se que a experiência, o conhecimento do médico e o apoio dos sistemas de informação facilitam a consulta do processo do paciente e o seu tratamento e, por isso, mesmo que busque informação na internet, é fundamental que o paciente se comunique com o médico e partilhe as dúvidas e as informações encontradas. Assim, com o recurso às tecnologias de informação, a comunicação em saúde é facilitada, permitindo um maior envolvimento do cidadão com seu médico, uma melhoria no acesso, um maior monitoramento e a promoção da auto-gestão da saúde e da doença.

\section{Referências}

1. Haug M. Re-examination of the hypothesis of physician deprofissionalization. Millbank Q. 1988; 66(Suppl 2):S48-65.

2. World Health Organization. Connecting for health: global vision, local insight. Genebra; 2005.

3. Fallows D. How women and men use the Internet [Internet]. Washington: Pew Internet \& Am Life Project; 2005 [cited 2018 May 21]. Available from: https://goo.gl/Lza3w9

4. Fox S. Health information online: eight in ten internet users have looked for health information online, with increased interest in diet, fitness, drugs, health insurance, experimental treatments, and particular doctors and hospitals [Internet]. Washington: Pew Internet \& Am Life Project; 2005 [cited 2018 May 21]. Available from: https://goo.gl/aAZaKS

5. Rice R. Influences, usage, and outcomes of Internet health information searching: multivariate results from them pew surveys. Int J Med Inform [Internet]. 2006 [cited 2018 May 21];75(1):8-28. doi: https://doi. org/10.1016/j.jimedinf.2005.07.032

6. Gilliam AD, Speake WJ, Scholefield JH, Beckingham IJ. Finding the best from the rest: evaluation of the quality of patient information on the Internet. Ann R Coll Surg Engl [Internet]. 2003 [cited 2018 May 21];85(1):44-6. Available from: https://goo.gl/HpLH3P

7. Iverson S, Howard K, Penney B. Impact of internet use on health-related behaviors and the patientphysician relationship: a survey-based study and review. J Am Osteopath Assoc [Internet]. 2008 [cited 2018 May 21];108(12):699-711. Available from: https://goo.gl/XwmqM9

8. Dutta-Bergman MJ. Health attitudes, health cognitions, and health behaviors among Internet health information seekers: population-based survey. J Med Internet Res [Internet]. 2004 [cited 2018 May 21];6(2):1-15. doi: https://doi.org/10.2196/jmir.6.2.e15

9. Hummel GS. ePatient: a odisseia digital do paciente em busca da saúde. São Paulo: Sts publicações; 2008.

10. Marschollek M, Mix S, Wolf KH, Effertz B, Haux R, Steinhagen-Thiessen E. ICT-based health information services for elderly people: past experiences, current trends, and future strategies. Med Inform Internet Med. 2007;32(4):251-61. doi: https://doi.org/10.1080/14639230701692736

11. SI.VID̄A - SI de Controlo do HIV / SIDA [Internet]. Lisboa: Serviços Partilhados do Ministério da Saúde; 2018 [citado em 2017 out. 20]. Disponível em: http://spms.min-saude.pt/product/sivida/

12. SINAVE - Sistema Nacional de Apoio à Vigilância Epidemiológica [Internet]. Lisboa: Serviços Partilhados do Ministério da Saúde; 2018 [citado em 2017 out. 20]. Disponível em: http://spms.minsaude.pt/product/sinave/

13. Jaspers K. II Medico nell'età della tecnica. Milano: Raffaello Cortina Editore; 1991.

14. Coelho Filho JM. Relação médico-paciente: a essência perdida. Interface (Botucatu) [Internet]. 2007;2(23):631-3. doi: http://dx.doi.org/10.1590/S1414-32832007000300018

15. Godfrey ME, Johnson O. Digital circles of support: meeting the information needs of older adults. Comput Hum Behav [Internet]. 2008 [cited 2018 May 21];25:633-42. doi: https://doi.org/10.1016/j. chb.2008.08.016

16. Goodman KW. Ethics, information technology, and public health: new challenges for the clinicianpatient relationship. J Law Med Ethics [Internet]. 2010 [cited 2018 May 21];38(1):58-63. doi: https://doi. org/10.1111/j.1748-720X.2010.00466.x

17. Observatório Português da Comunicação. A internet em Portugal: sociedade em rede 2014 [Internet]. Lisboa; 2014 [citado em 2018 maio 21]. Disponível em: https://obercom.pt/wp-content/uploads/2016/06/ A-Internet-em-Portugal-Sociedade-em-Rede-2014.pdf 
18. Ritterband L, Borowitz S, Cox D, Kovatchev B, Walker L, Lucas V, et al. Using the Internet to provide information prescriptions. Pediatrics [Internet]. 2005 [cited 2018 May 21];116(5):643-7. Available from: http://pediatrics.aappublications.org/content/116/5/e643.long

19. Kim J, Kim S. Physicians perception of the effects of Internet health information on the doctor-patient relationship. Inform Health Soc Care [Internet]. 2009 [cited 2018 May 21];34(3):136-48. doi: https://doi. org/10.1080/17538150903102422

20. Santana S, Pereira AS. Da utilização da internet para questões de saúde e doença em Portugal: possíveis repercussões na relação médico-paciente. Acta Med Port [Internet]. 2007 [citado em 2018 maio 21];20(1):47-57. Disponível em: https://goo.gl/cU9rAz

21. Alves N. Perfis dos utilizadores da internet em Portugal. Anál Social [Internet]. 2008 [citado em 2018 maio 21];43(188):603-25. Disponível em: http://www.scielo.mec.pt/pdf/aso/n188/n188a06.pdf

22. Masi C, Cassey M, Suarez-Balcazar Y, Cassey MZ, Kinney L, Piotrowski H . Internet access and empowerment: a community-based health initiative. J Gen Intern Med [Internet]. 2003 [cited 2018 May 21];18(7):525-30. Available from: https://goo.gl/Na3cMX

23. Stevenson F, Kerr C, Murray E, Nazareth I. Information from the Internet and the doctornpatient relationship: the patient perspective: a qualitative study. BMC Fam Pract [Internet]. 2007 [cited 2018 May 21];8(47):1-8. doi: https://doi.org/10.1186/1471-2296-8-47

24. Walker JM, Carayon, P. From tasks to processes: the case for changing health information technology to improve health care. Health Aff (Millwood). 2009 [cited 2018 May 21];28(2):467-77. doi: https://doi. org/10.1377/hlthaff.28.2.467

25. Bardin L. Análise de conteúdo. Lisboa: Edições 70; 2013.

26. Amatal A, Valle A, Monat A, Marques E. Sistemas de informações gerenciais em organizações de saúde. Rio de Janeiro: FGV; 2010.

27. Macgregor R, Hyland P, Harvie C. Associations between driving forces to adopt ICT and benefits derived from that adoption in medical practices in Australia. In: Handbook of research on developments in e-health and telemedicine: technological and social perspectives. IGI Global; 2010:652-668.

28. El Sayed H, Westrup C. Egypt and ICTs: how ICTs bring national initiatives, global organizations and local companies together. Inf technol people [Internet]. 2003 [cited 2018 May 21];16(1):76-92. doi: https://doi.org/10.1108/09593840310463041

29. Akesson K, Saveman B, Nilssom G. Health care consumers' experiences of information communication technology: a summary of literature. Int J Med Inform [Internet]. 2007 [cited 2018 May 21];76(9):633-45. doi: https://doi.org/10.1016/j.jimedinf.2006.07.001

30. Seckin G. Patients as information managers: the internet for successful self-health care \& illness management. Open Longev Sci [Internet]. 2010 [cited 2018 May 21];4:36-42. Available from: https://goo. $\mathrm{gl} / \mathrm{YfsgC1}$

31. Salles A. Transformações na relação médico-paciente na era da informatização. Rev Bioét [Internet]. 2010 [citado em 2018 maio 21];18(1):49-60. Disponível em: https://goo.gl/XoeXaz

32. Pereira Neto A, Guilam M. The Internet, expert patients and medical practice: an analysis of the literature. Interface (Botucatu) [Internet]. 2008 [cited 2018 May 21];12(26):579-88. doi: http://dx.doi. org/10.1590/S1414-32832008000300010

33. Grosseman S, Stoll C. O ensino-aprendizagem da relação médico-paciente: estudo de caso com estudantes do último semestre do curso de medicina. Rev Bras Educ Med [Internet]. 2008 [citado em 2018 maio 21];32(3):301-8. doi: http://dx.doi.org/10.1590/S0100-55022008000300004

34. Budtz S, Witt K. Consulting the Internet before visit to general practice. Patients' use of the Internet and other sources of health information. Scand J Prim Health Care [Internet]. 2002 [cited 2018 May 21];20(3):174-6. doi: https://doi.org/10.1080/028134302760234645

35. Grover F, Wu H, Blanford C, Holcomb S, Tidler D. Computer-using patients want Internet services from family physicians. J Fam Pract. 2002;51(6):570-2.

36. Diaz JA, Griffith RA, Ng JJ, Reinert SE, Friedmann PD, Moulton AW. Patients' use of the Internet for medical information. J Gen Intern Med [Internet]. 2002 [cited 2018 May 21];17(3):180-5. doi: https:// dx.doi.org/10.1046\%2Fj.1525-1497.2002.10603.x 
37. Andreassen HK, Bujnowska-Fedak MM, Chronaki CE, Dumitru RX, Pudule I, Santana S, et al. European citizens' use of Ehealth services: a study of seven countries. BMC Public Health [Internet]. 2007 [cited 2018 May 21];7:53-9. Available from: https://goo.gl/KU1eEv

38. Fox S, Rainie L. Pew internet and american life project. "Vital decisions", summary of findings, and part six: impact. Washington; 2002.

39. Henwood F, Wyatt S, Hart A, Smith J. Ignorance is a bliss sometimes: constraints on the emergence of the "informed patient" in the changing landscape of health information. Sociol. Health Illn [Internet]. 2003 [cited 2018 May 21];25(6):589-607. doi: https://doi.org/10.1111/1467-9566.00360

40. Pandey S, Hart J, Tiwary S. Women's health and the internet: understanding emerging trends and implications. Soc Sci Med [Internet]. 2003 [cited 2018 May 21];56(1):179-91. doi: https://doi. org/10.1016/S0277-9536(02)00019-9

41. Skinner H, Biscope S, Poland B. Quality of internet access: barrier behind internet use statistics. Soc. Sci. Med [Internet]. 2003 [cited 2018 May 21];57(5):875-80. doi: https://doi.org/10.1016/S02779536(02)00455-0

42. Cotten S, Gupta S. Characteristics of online and offline health information seekers and factors that discriminate between them. Soc Sci Med [Internet]. 2004 [cited 2018 May 21];59(9):1795-806. doi: https://doi.org/10.1016/j.socscimed.2004.02.020

43. Berger M, Wagner T, Baker L. Internet use and stigmatized illness. Soc Sci Med [Internet]. 2005 [cited 2018 May 21];61(8):1821-7. doi: https://doi.org/10.1016/j.socscimed.2005.03.025

44. Nettleton S, Burrows R, O'Malley L. The mundane realities of the everyday lay use of the internet for health, and their consequences for media convergence. Sociol Health Illn [Internet]. 2005 [cited 2018 May 21];27(7):972-92. doi: https://doi.org/10.1111/j.1467-9566.2005.00466.x

45. Hardey M. Doctor in the house: the internet as a source of lay health knowledge and the challenge to expertise. Sociol. Health Illn [Internet]. 1999 [cited 2018 May 21];21(6):820-35. doi: https://doi. org/10.1111/1467-9566.00185

46. Drentea P, Moren-Cross J. Social capital and social support on the web: the case of an internet mother site. Sociol. Health Illn [Internet]. 2005 [cited 2018 May 21];27(7):920-43. doi: https://doi.org/10.1111/ j.1467-9566.2005.00464.x

47. Fox N, Ward K, O'Rourke A. The 'expert patient': empowerment or medical dominance? The case of weight loss, pharmaceutical drugs and the internet. Soc Sci Med [Internet]. 2005 [cited 2018 May 21];60(6):1299-309. doi: https://doi.org/10.1016/j.socscimed.2004.07.005

48. Ziebland $\mathrm{S}$. The importance of being expert: the quest for cancer information on the internet. Soc Sci Med [Internet]. 2004 [cited 2018 May 21];59(9):1783-93. doi: https://doi.org/10.1016/j. socscimed.2004.02.019 